\title{
Heat Flux at the Surface of Metal Foil Heater under Evaporating Sessile Droplets
}

\author{
Igor Marchuk, ${ }^{1,2}$ Andrey Karchevsky, ${ }^{1,3}$ Anton Surtaev, ${ }^{2}$ and Oleg Kabov ${ }^{2,4}$ \\ ${ }^{1}$ Novosibirsk State University, Novosibirsk 630090, Russia \\ ${ }^{2}$ Kutateladze Institute of Thermophysics SB RAS, Novosibirsk 630090, Russia \\ ${ }^{3}$ Sobolev Institute of Mathematics SB RAS, Novosibirsk 630090, Russia \\ ${ }^{4}$ Tomsk Polytechnic University, Tomsk 634050, Russia
}

Correspondence should be addressed to Igor Marchuk; marchuk@itp.nsc.ru

Received 6 September 2015; Accepted 10 November 2015

Academic Editor: Corin Segal

Copyright (C) 2015 Igor Marchuk et al. This is an open access article distributed under the Creative Commons Attribution License, which permits unrestricted use, distribution, and reproduction in any medium, provided the original work is properly cited.

\begin{abstract}
Evaporating water drops on a horizontal heated substrate were investigated experimentally. The heater was made of a constantan foil with the thickness of $25 \mu \mathrm{m}$ and size of $42 \times 35 \mathrm{~mm}^{2}$. The temperature of the bottom foil surface was measured by the infrared (IR) camera. To determine the heat flux density during evaporation of liquid near the contact line, the Cauchy problem for the heat equation was solved using the temperature data. The maximum heat flux density is obtained in the contact line region and exceeds the average heat flux density from the entire foil surface by the factor of 5-7. The average heat flux density in the region wetted by the drop exceeds the average heat flux density from the entire foil surface by the factor of 3-5. This fact is explained by the heat influx from the foil periphery to the drop due to the relatively high heat conductivity coefficient of the foil material and high evaporation rate in the contact line region. Heat flux density profiles for pairs of sessile droplets are also investigated.
\end{abstract}

\section{Introduction}

Liquid droplets moving or spreading on the solid surface are widely distributed in nature, different areas of techniques, and technology. For example, they are observed in the coating technologies of solid surfaces by liquid films, during spraying of fertilizers and pesticides, in strengthening steel devices, in the spray cooling systems for electronic equipment, and others. Flow regime of liquid droplets is main in a two-phase mixture in rectangular channels of small height as shown in [1]. The angle between the solid surface and the tangent to the drop surface at the point of contact between the three phases (wetting angle) is a fundamental macroscopic characteristic of the contact line. This angle is determined by the equation of Young [2], as a result of the mechanical balance of the three surface tensions: liquid-gas, solid surface-gas, and solid surface-liquid. In the literature significant attention is paid to the study of various aspects of the droplets spreading over the solid surface. Only a few studies [2-8] have been performed for conditions with different level of gravity. Analysis of these papers shows that there is lack of understanding of the process of evaporation and wetting by droplets over solid surfaces in condition of changing gravity. Wetting of solid surface by droplets and processes in three-phase contact line gas-liquid-solid has a major influence on the droplets evaporation. Insufficient understanding of the properties of the three-phase contact line is currently holding back the development of mathematical models of evaporation of liquid droplets on solid surfaces. On the other hand, the liquid droplet on a solid surface is the most simple and convenient object for studying phenomena of wetting and spreading and properties of the three-phase contact line. In particular process of evaporation in the three-phase contact line can be studied on droplets. The authors along with research groups from Europe, Canada, China, and Japan are members in the preparation of the experiment "Drop Evaporation" on board the International Space Station.

The transfer processes in the vicinity of a dynamic threephase wall-liquid-vapor contact line belong to one of the most important physical problems not yet fully solved. Insufficient 
understanding of transfer processes in the region of the threephase contact line impedes the development of models of several phenomena, such as boiling, cavitation, dropwise condensation, rivulet flows, liquid film break, drop spreading, and evaporation [9-11]. The liquid thickness in this zone with the length of about several microns, which is often called the microregion, decreases from $1-3 \mu \mathrm{m}$ to $10-20 \mathrm{~nm}$ (the adsorbed film). Significant curvature of the gas-liquid interface in the microregion, change in the thickness by 2-3 orders of magnitude, nonequilibrium effects (the temperature drop at the interface), influence of van der Waals forces, and structure of the substrate cause considerable difficulties for both experimental and theoretical investigations of this configuration.

The problem of determining the evaporation rate and heat flux density in the heated liquid in the three-phase contact line region is actively studied in connection with important practical applications in power engineering, medicine, and chemical, pharmaceutical, and food industries [12-14]. Both theoretical and experimental studies prove that heat transfer intensity in the contact line region can be more than an order of magnitude higher than the average one and it causes a local minimum in the temperature profile along the solid wall $[15,16]$. However, since it is impossible to perform the direct measurements of the heat flux in the microregion (the length of which is estimated to be between 0.5 and 10$20 \mu \mathrm{m}$ ), various indirect methods and numerical procedures are applied. In Ibrahem et al. [17], the meniscus of HFE7100 liquid evaporated between two heated vertical plates of metal foil. The local heat flux to evaporating meniscus was calculated using the two-dimensional temperature distribution along the outer foil edge, obtained by the infrared scanner with resolution of 14.8 microns. It was found that the local heat flux density in the contact line region was 5.4-6.5 times higher than the average heat flux density on the surface.

In the present study, evaporation near contact lines is investigated using the sessile drop configuration. There is a significant amount of literature on evaporating sessile droplets $[9,11,16]$. However, the use of metal foil as a heater in droplet experiments is not common, despite the fact that such configuration has potential for giving accurate measurements of the temperature field immediately under the droplet and thus providing a tool for studies of local coupling of the triple line motion and heat transfer in the substrate with unprecedented accuracy. We believe that this potential has not been fully realized. For example, Sodtke et al. [16] used a thin metal foil heater but relied on thermochromic liquid crystals (TLCs) for temperature measurement. The issues with TLCs are limited accuracy and lack of applicability outside of a relatively narrow temperature range. The objective of the present study is to overcome these difficulties by using IR imaging. Another limitation of the current literature on droplets is that the vast majority of both theoretical and experimental studies are focused on isolated droplets, while in applications one often encounters large arrays of droplets. The issue of interaction of droplets remains poorly understood and is discussed in the present study.

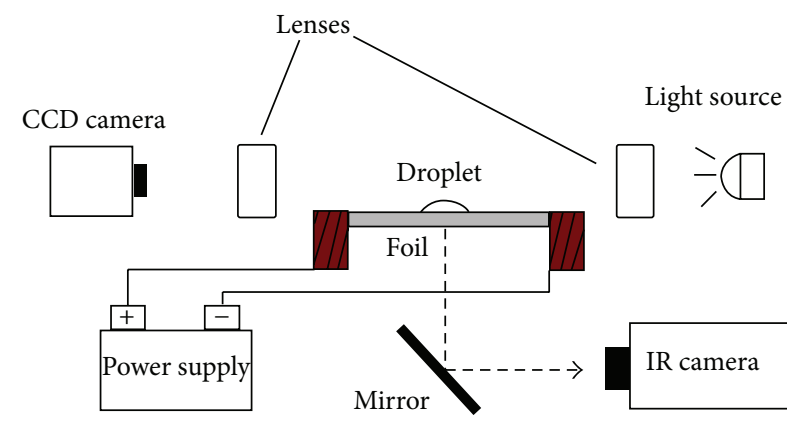

FIGURE 1: The scheme of the experiment.

\section{Materials and Methods}

2.1. Experiment. The evaporating sessile water drops on a horizontal heated substrate were studied experimentally using the setup shown in Figure 1. The constantan foil $(\mathrm{CuNi})$ of the thickness of $25 \mu \mathrm{m}$, size of $42 \times 35 \mathrm{~mm}^{2}$ $(A \times B)$, and heat conductivity $\lambda$ of $23 \mathrm{~W} / \mathrm{mK}$ was used as the substrate. The substrate was heated by Joule heating. In experiments, the heating power $Q$ varied from $0.25 \mathrm{~W}$ to $2.5 \mathrm{~W}$. The images of drop profile were obtained by the optical shadow system with resolution of $8 \mu \mathrm{m} / \mathrm{px}$. The geometric parameters of drops were determined using the images by the Young-Laplace method; in experiments they were as follows: the volume was $5.1-39.3 \mu \mathrm{L}$, base diameter (wetted spot) was $3.68-7.19 \mathrm{~mm}$, height was $0.88-1.81 \mathrm{~mm}$, and contact angle was $50-75^{\circ}$ (average angle of $63^{\circ}$ ). The temperature of the bottom foil surface was measured by IR camera Titanium 570M. The thermographic study was performed at recording frequency of $25 \mathrm{~Hz}$, resolution of $640 \times 512$ pixels $(108 \mu \mathrm{m} / \mathrm{px})$, and integration time of $805 \mathrm{~ms}$. To increase the minimal resolvable temperature difference when using the IR cameras, the reverse side of the foil was coated with a soot layer. The foil surface temperature measured in experiments was in the range of $29-86^{\circ} \mathrm{C}$. The average coefficient of heat transfer from the foil surface to surrounding air $\alpha_{\text {av }}$ was found to be within $16-20 \mathrm{~W} / \mathrm{m}^{2} \mathrm{~K}$, as seen in Figure 2. These measurements were performed without droplets on the foil surface.

2.2. Theory and Computation. To obtain accurate description of heat transfer intensity near the contact line for evaporating sessile droplets, the Cauchy problem was formulated for the elliptic equation

$$
\lambda \Delta T+q_{v}=0,
$$

describing the heat conduction in the foil (i.e., the domain defined by the equations $A / 2 \leq x \leq A / 2, B / 2 \leq y \leq$ $B / 2$, and $0 \leq z \leq H)$. Here $T(x, y, z)$ is temperature of the foil, $\lambda$ is (constant) thermal conductivity, and $q_{v}$ is the constant volume heat source. On the bottom side of the foil, the temperature and the heat flux density were set as

$$
\begin{gathered}
T(x, y, 0)=T_{w}(x, y), \\
\lambda \frac{\partial T}{\partial z}(x, y, 0)=\alpha_{\mathrm{av}}\left(T_{w}-T_{a}\right) .
\end{gathered}
$$




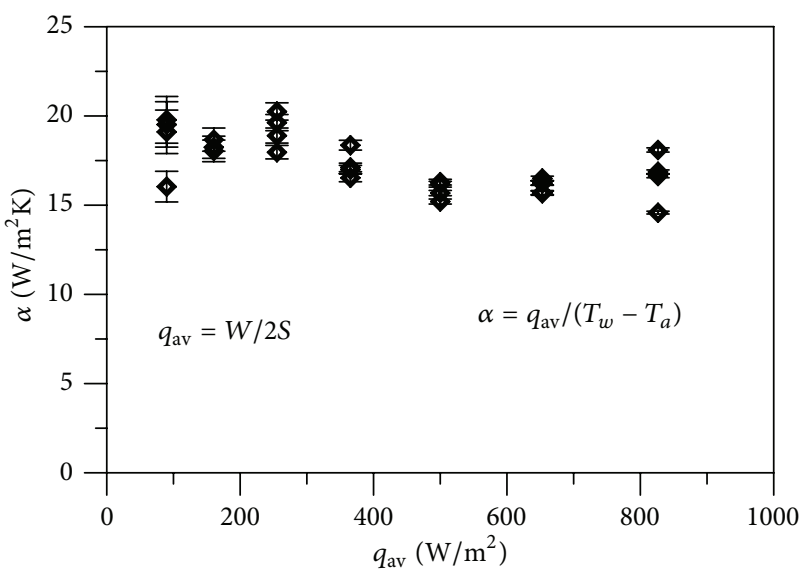

FIGURE 2: The measured heat transfer coefficient for different heat flux densities. $S$ : area of one side of the foil, $W$ : power of electric heating of the foil, $T_{w}$ : average measured temperature of the foil surface, and $T_{a}$ : temperature of ambient air.

The lateral walls were assumed adiabatic:

$$
\left.\frac{\partial T}{\partial x}\right|_{x= \pm A / 2}=\left.\frac{\partial T}{\partial y}\right|_{y= \pm B / 2}=0 .
$$

The Cauchy problem for the Laplace equation or elliptic equations of the general form is one of the oldest ill-posed problems [18-22]. Its solution is unstable: small variations in the Cauchy data can lead to significant changes in the solution. If the existence of a bounded solution assumed, it is possible to show the conditional stability of the problem [20]. There are many different implementations of methods of numerical solution to the Cauchy problem for elliptic equations (see, e.g., [21-25]). Instability of solution to the Cauchy problem becomes obvious, when applying any of numerical methods to its solution. In this work, we use the method that reduces the Cauchy problem solution to the solution of the problem of moments $[24,25]$. By a special choice of the boundary conditions in the conjugated problem, calculation of the heat flux value at the boundary part, inaccessible for the measurements, is reduced to summation of the corresponding series. Simple form of the domain allows solving the moment problem using the direct and inverse Fourier transforms. The regularizing procedure is used to sum the series of inverse Fourier transforms due to the instability of the Cauchy problem. The implemented method of solution was applied to experimental data processing.

\section{Results and Discussion}

With the use of the above method for solving the Cauchy problem, distributions of heat flux density on the foil surface from the drop side were obtained from the thermographic measurements. The initially measured temperature field, calculated distribution of heat flux density on the substrate, and distribution of the local heat flux density along the line passing through the central cross section of the drop are shown in Figure 3. The drop profile is also shown there. The results show that the maximum of the heat flux density is in the contact line region and it exceeds the average heat flux density on the entire foil surface by the factor of 5-7. The average heat flux in the zone, wetted by a drop, exceeds the average heat flux density on the entire foil surface by the factor of 3-5. This can be explained by the heat influx from the foil periphery to the drop due to the relatively high heat conductivity coefficient of the foil material and high evaporation rate in the contact line region. Calculation of local heat flux density distribution without considering redistribution of heat released in foil (line 2) is also shown in Figure 3. It can be seen that a negligence of substrate heat conductivity gives significantly underestimated heat flux density and absence of peaks in the contact line region. Data are shown for two different sizes of drops at substantially different average heat flux density. It can be seen that the ratio of maximal heat flux density in the contact line region and average heat flux density increases with a drop size decrease; it is equal to 3.7 for Figure 3(a) and 4.8 for Figure 3(b).

Previous results suggest that the heat flow from the dry part of the foil to the droplet plays an important role in the overall heat transfer, suggesting that evaporation rates for two or more droplets can be significantly different from the values predicted for a single droplet. The results of measurements and calculations for two sessile drops on the substrate are shown in Figure 4; the distance between these drops is $1.1 \mathrm{~mm}$. The symmetry conditions are broken for each of these drops. In the contact line region between drops we do not observe the maxima of heat flux density because the heat influx from the periphery, which is now split between the two drops, decreases for each of these drops. The maxima of heat flux density are reached near the contact line at the outer edges of the two-droplet system; the maximum value is higher for the smaller droplet.

The droplet on the heated substrate evaporates with time. The evaporation rate can be evaluated using the available data through the heat flux density:

$$
q_{\mathrm{ev}}=q_{t} \cos (\beta)-q_{\mathrm{conv}} .
$$

Here, $q_{t}$ is the calculated heat flux density on the substrate, $\beta$ is an angle of inclination of the drop surface to horizontal substrate, and $q_{\text {conv }}$ is a convective heat flux from the droplet surface:

$$
q_{\mathrm{conv}}=\alpha\left(T_{s}-T_{a}\right)
$$

It can be expressed through $q_{b}$, which is the heat flux from the lower foil surface in the assumption that the temperature of droplet surface slightly differs from the temperature of substrate under it; $T_{s} \cong T_{b}$. At that, we should take into account the difference between the area of droplet surface and substrate:

$$
q_{\mathrm{conv}}=q_{b} \cos (\beta) .
$$

We obtain

$$
q_{\mathrm{ev}}=\left(q_{t}-q_{b}\right) \cos (\beta) .
$$



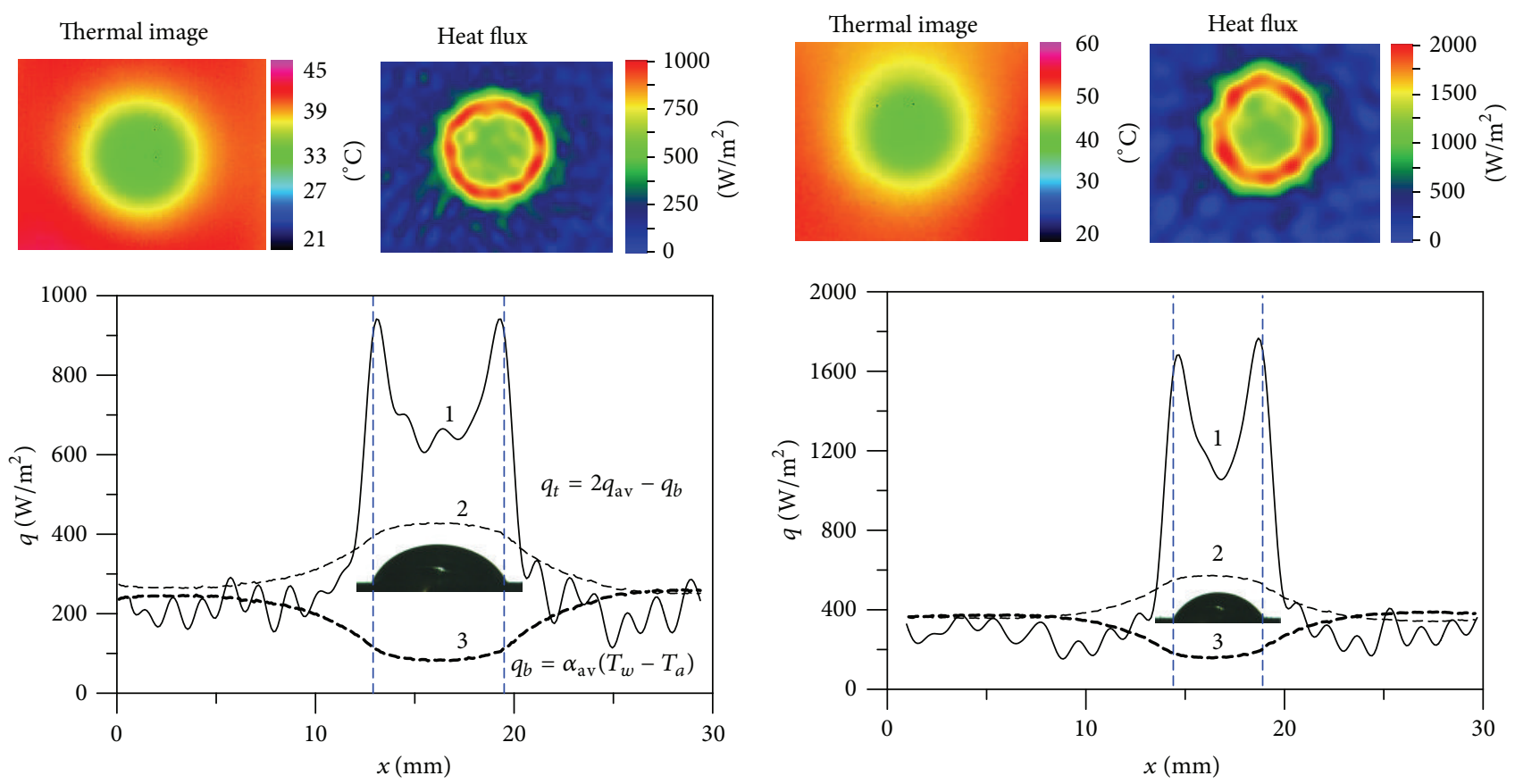

(a)

(b)

Figure 3: Distributions of heat flux density: 1: Cauchy problem solution. 2: calculation with neglecting of heat flux redistribution in the foil, $q_{t}$. 3: on the bottom side of foil, $q_{b}$. (a) Drop $D=6.6 \mathrm{~mm}, H=1.82 \mathrm{~mm}, V=36.3 \mu \mathrm{L}, \theta=65^{\circ}$, and $q_{\mathrm{av}}=255 \mathrm{~W} / \mathrm{m}^{2}$. (b) Drop $D=4.4 \mathrm{~mm}$, $H=1.15 \mathrm{~mm}, V=9.8 \mu \mathrm{L}, \theta=59^{\circ}$, and $q_{\mathrm{av}}=365 \mathrm{~W} / \mathrm{m}^{2}$.
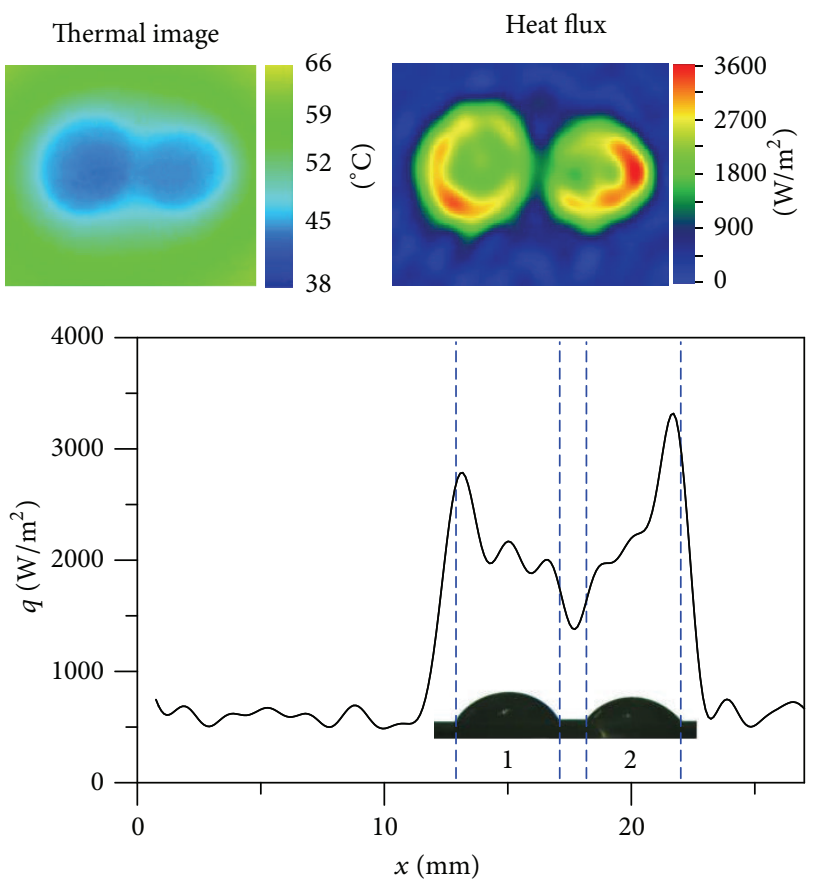

FIGURE 4: Distribution of heat flux density based on Cauchy problem solution for two drops, $q_{\mathrm{av}}=653 \mathrm{~W} / \mathrm{m}^{2}:$ (1) $D=4.1 \mathrm{~mm}, H=$ $1.085 \mathrm{~mm}, V=7 \mu \mathrm{L}$, and $\theta=58^{\circ}$; (2) $D=4.1 \mathrm{~mm}, H=0.903 \mathrm{~mm}$, $V=5.73 \mu \mathrm{L}$, and $\theta=52^{\circ}$.
The local evaporation rate can be calculated as

$$
\dot{m}=\frac{q_{\mathrm{ev}}}{r_{\mathrm{lv}}}=\frac{\left(q_{t}-q_{b}\right) \cos (\beta)}{r_{\mathrm{lv}}},
$$

where $r_{\mathrm{lv}}$ is the latent heat of vaporization. Evaporation heat flux density and evaporation rate for the droplets of different diameters are shown in Figure 5. It can be seen that evaporation rate near the contact line has a local maximum, but it is not so pronounced as heat flux density on the substrate in Figures 3 and 4.

\section{Conclusions}

We have shown that the maximum of the heat flux density is in the contact line region and it exceeds the average heat flux density on the entire foil surface by the factor of 57. In two-droplet system the maxima of heat flux density are reached near the contact line at the outer edges of the two-droplet system; the maximum value is higher for the smaller droplet. This can be explained by the heat influx from the foil periphery to the drop due to the relatively high heat conductivity coefficient of the foil material and high evaporation rate in the contact line region. The possibility of heat flux calculation by using infrared data gives interesting method of an experimental study of three-phase contact line. 


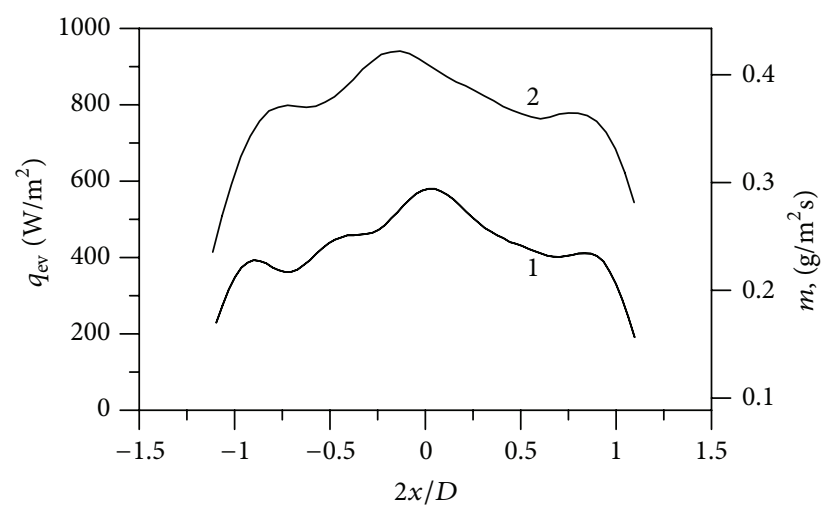

FIGURE 5: Calculated distribution of evaporation heat flux density and evaporation rate: (1) drop from Figure 3(a) and (2) drop from Figure 3(b).

\section{Conflict of Interests}

The authors declare that there is no conflict of interests regarding the publication of this paper.

\section{Acknowledgment}

The study was financially supported by the grant of the Russian Science Foundation (Project no. 14-39-00003).

\section{References}

[1] E. A. Chinnov and O. A. Kabov, "Two-phase flows in horizontal flat microchannels," Doklady Physics, vol. 57, no. 1, pp. 42-46, 2012.

[2] A. Ababneh, A. Amirfazli, and J. A. W. Elliott, "Effect of gravity on the macroscopic advancing contact angle of sessile drops," Canadian Journal of Chemical Engineering, vol. 84, no. 1, pp. 3943, 2006.

[3] D. Brutin, Z. Zhu, O. Rahli, J. C. Xie, Q. S. Liu, and L. Tadrist, "Sessile drop in microgravity: creation, contact angle and interface," Microgravity Science and Technology, vol. 21, no. 1, pp. 67-76, 2009.

[4] G. Abel, G. G. Ross, and L. Andrzejewski, "Wetting of a liquid surface by another immiscible liquid in microgravity," Advances in Space Research, vol. 33, no. 8, pp. 1431-1438, 2004.

[5] D. Brutin, Z.-Q. Zhu, O. Rahli, J.-C. Xie, Q.-S. Liu, and L. Tadrist, "Evaporation of ethanol drops on a heated substrate under microgravity conditions," Microgravity Science and Technology, vol. 22, no. 3, pp. 387-395, 2010.

[6] F. Carle, B. Sobac, and D. Brutin, "Hydrothermal waves on ethanol droplets evaporating under terrestrial and reduced gravity levels," Journal of Fluid Mechanics, vol. 712, pp. 614-623, 2012.

[7] O. A. Kabov and D. V. Zaitsev, "The effect of wetting hysteresis on drop spreading under gravity," Doklady Physics, vol. 58, no. 7, pp. 292-295, 2013.

[8] F. Carle, B. Sobac, and D. Brutin, "Experimental evidence of the atmospheric convective transport contribution to sessile droplet evaporation," Applied Physics Letters, vol. 102, no. 6, Article ID 061603, 2013.
[9] V. K. Dhir, "Numerical simulations of pool-boiling heat transfer," AIChE Journal, vol. 47, no. 4, pp. 813-834, 2001.

[10] R. J. Braun, "Dynamics of the tear films," Annual Review of Fluid Mechanics, vol. 44, pp. 267-297, 2012.

[11] B. Sobac and D. Brutin, "Triple-line behavior and wettability controlled by nanocoated substrates: influence on sessile drop evaporation," Langmuir, vol. 27, no. 24, pp. 14999-15007, 2011.

[12] C. L. Moraila-Martínez, M. A. Cabrerizo-Vílchez, and M. A. Rodríguez-Valverde, "Controlling the morphology of ring-like deposits by varying the pinning time of driven receding contact lines," Interfacial Phenomena and Heat Transfer, vol. 1, no. 1, pp. 195-205, 2013.

[13] A. Kundan, J. L. Plawsky, and P. C. Wayner Jr., “Thermophysical characteristics of a wickless heat pipe in microgravityconstrained vapor bubble experiment," International Journal of Heat and Mass Transfer, vol. 78, pp. 1105-1113, 2014.

[14] V. S. Ajaev, E. Y. Gatapova, and O. A. Kabov, "Rupture of thin liquid films on structured surfaces," Physical Review E, vol. 84, no. 4, Article ID 041606, 2011.

[15] S. Moosman and G. M. Homsy, "Evaporating menisci of wetting fluids," Journal of Colloid and Interface Science, vol. 73, no. 1, pp. 212-223, 1980.

[16] C. Sodtke, V. S. Ajaev, and P. Stephan, "Dynamics of volatile liquid droplets on heated surfaces: theory versus experiment," Journal of Fluid Mechanics, vol. 610, pp. 343-362, 2008.

[17] K. Ibrahem, M. F. Abd Rabbo, T. Gambaryan-Roisman, and P. Stephan, "Experimental investigation of evaporative heat transfer characteristics at the 3-phase contact line," Experimental Thermal and Fluid Science, vol. 34, no. 8, pp. 1036-1041, 2010.

[18] J. Hadamard, Lectures on the Cauchy Problem in Linear Differential Equations, Yale University Press, New Haven, Conn, USA, 1923.

[19] L. E. Payne, "Bounds in the Cauchy problem for the Laplace equation," Archive for Rational Mechanics and Analysis, vol. 5, pp. 35-45, 1960.

[20] M. Lavrentev, V. Romanov, and S. Shishatskii, Ill-Posed Problems of Mathematical Physics and Analysis, American Mathematical Society, 1986.

[21] F. Berntsson and L. Eidén, "Numerical solution of a Cauchy problem for the Laplace equation," Inverse Problems, vol. 17, no. 4, pp. 839-853, 2001.

[22] L. Bourgeois and J. Dardé, "A duality-based method of quasireversibility to solve the Cauchy problem in the presence of noisy data," Inverse Problems, vol. 26, no. 9, Article ID 095016, 2010.

[23] C.-L. Fu, H.-F. Li, Z. Qian, and X.-T. Xiong, "Fourier regularization method for solving a Cauchy problem for the Laplace equation," Inverse Problems in Science and Engineering, vol. 16, no. 2, pp. 159-169, 2008.

[24] T. Regińska and A. Wakulicz, "Wavelet moment method for the Cauchy problem for the Helmholtz equation," Journal of Computational and Applied Mathematics, vol. 223, no. 1, pp. 218229, 2009.

[25] A. L. Karchevsky, "Reformulation of an inverse problem statement that reduces computational costs," Eurasian Journal of Mathematical and Computer Applications, vol. 1, no. 2, pp. 5-20, 2013. 

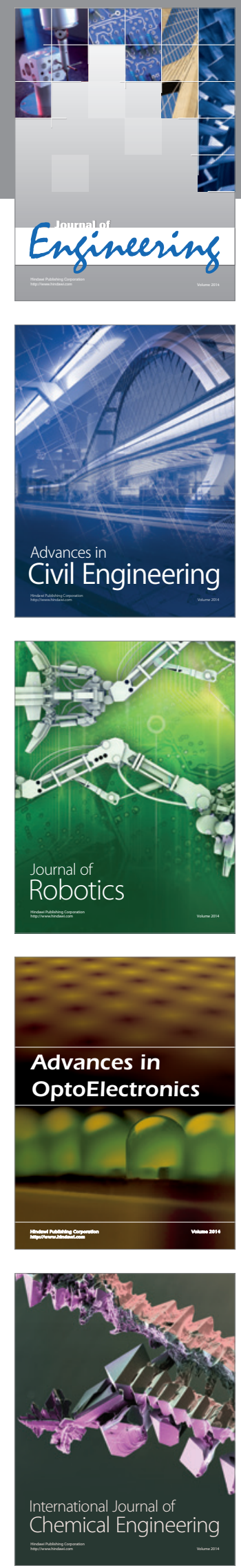

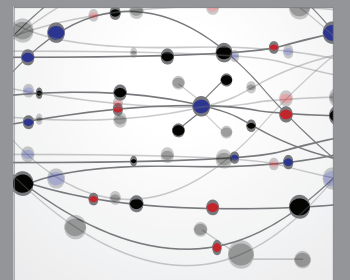

The Scientific World Journal
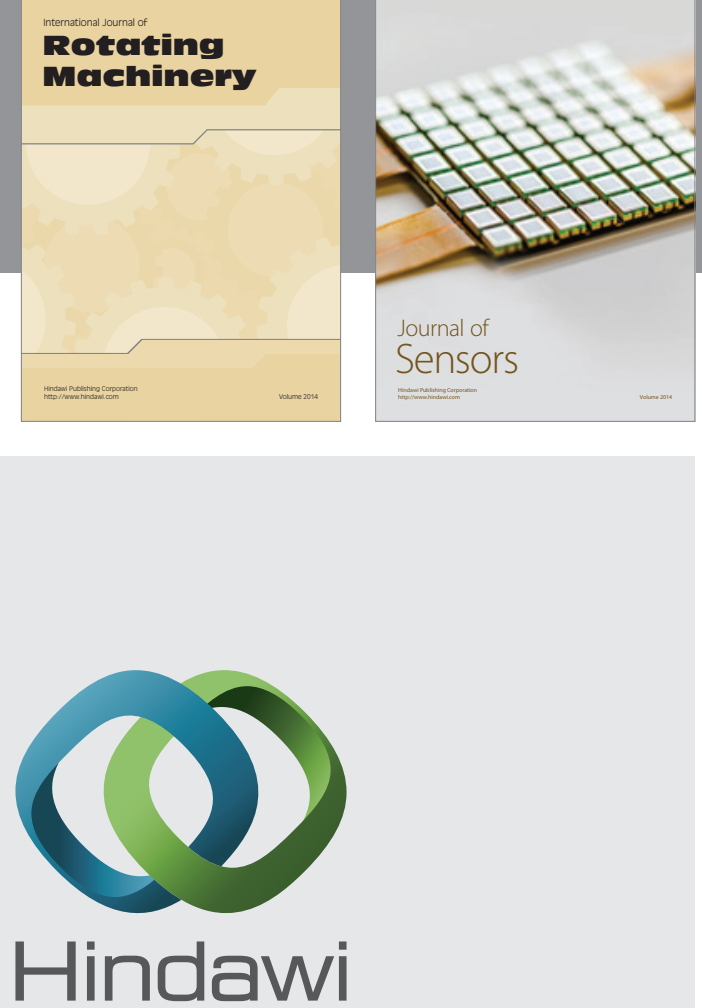

Submit your manuscripts at http://www.hindawi.com
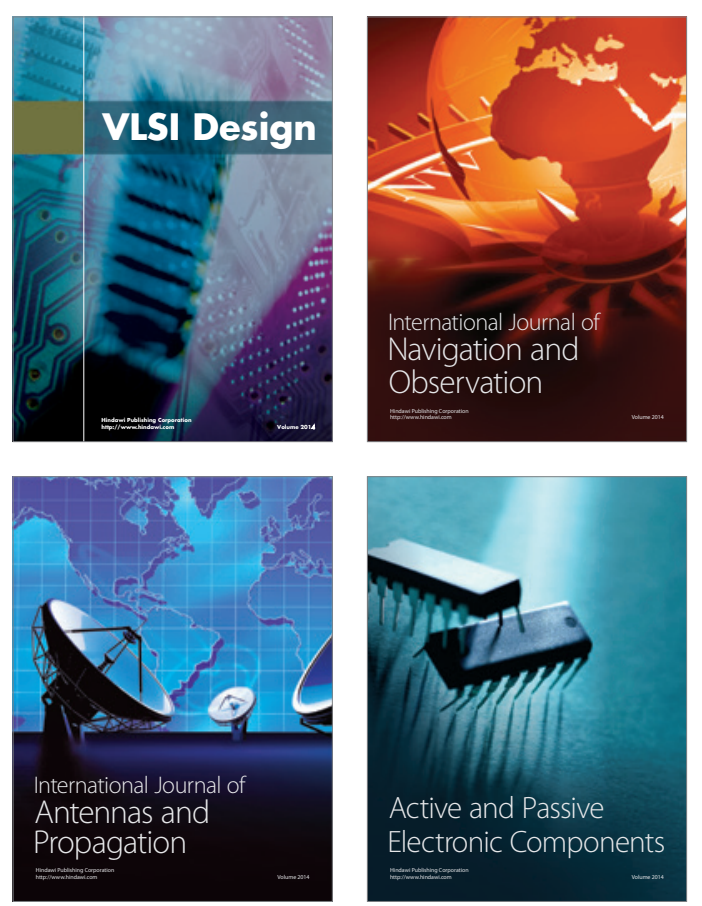
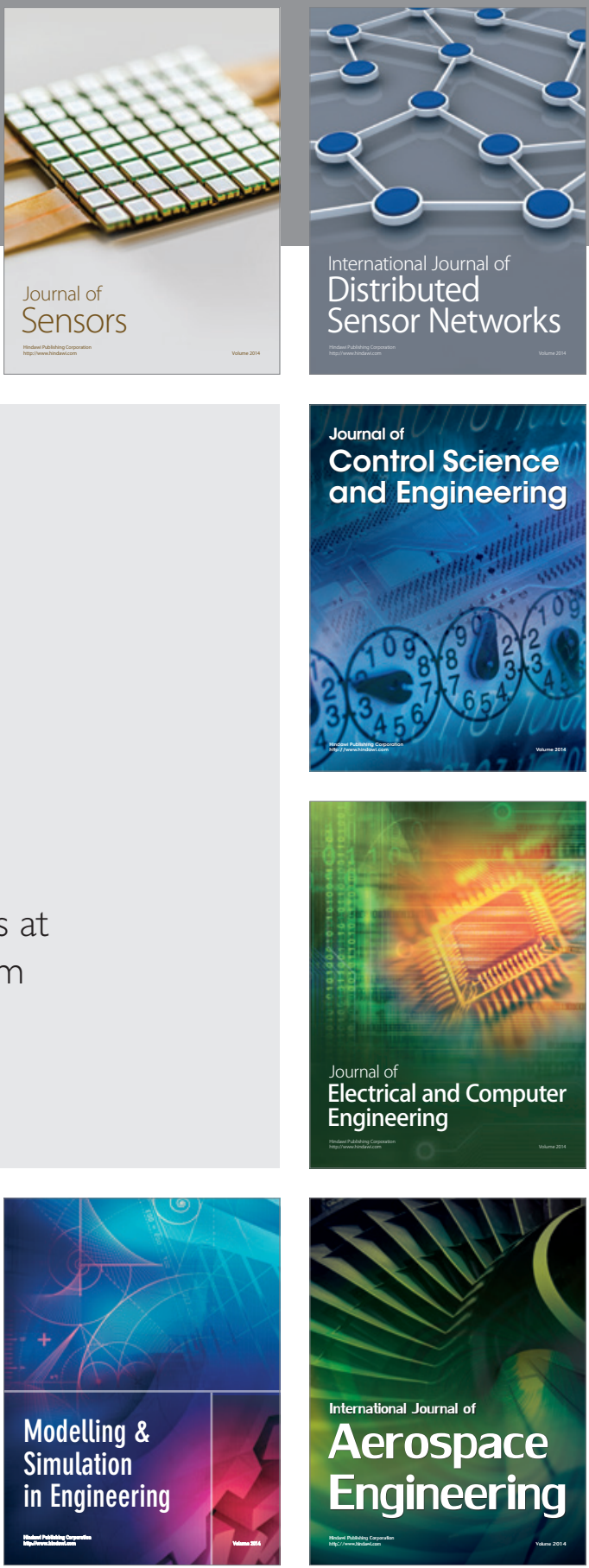

Journal of

Control Science

and Engineering
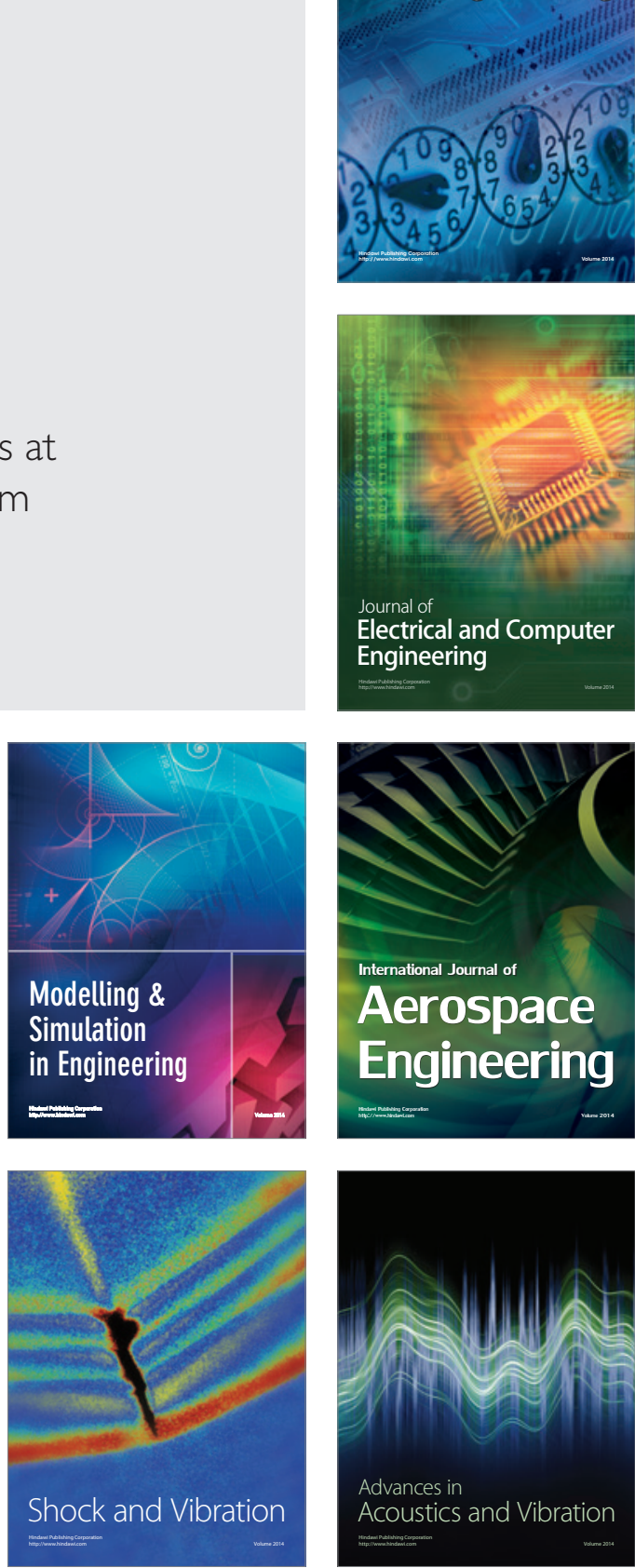